\title{
Weighted gene co-expression network analysis of colorectal cancer liver metastasis genome sequencing data and screening of anti-metastasis drugs
}

\author{
BO GAO $^{1}$, QIN SHAO ${ }^{2}$, HANI CHOUDHRY ${ }^{3}$, VICTORIA MARCUS ${ }^{2}$, \\ KUNG DONG $^{4}$, JIANNIS RAGOUSSIS ${ }^{3}$ and ZU-HUA GAO ${ }^{2}$
}

\author{
${ }^{1}$ Department of General Surgery, The First Affiliated Hospital of Harbin Medical University, Harbin, Heilongjiang 150001, \\ P.R. China; ${ }^{2}$ Department of Pathology, The Research Institute of McGill University Health Center, Montreal, \\ Québec H4A 3J1; ${ }^{3}$ McGill University and Genome Quebec Innovation Centre, Montreal, Québec H3B 1S6, Canada; \\ ${ }^{4}$ Department of Pathology, Beijing Youan Hospital, Capital Medical University, Beijing 100069, P.R. China
}

Received April 25, 2016; Accepted June 3, 2016

DOI: 10.3892/ijo.2016.3591

\begin{abstract}
Approximately 9\% of cancer-related deaths are caused by colorectal cancer (CRC). CRC patients are prone to liver metastasis, which is the most important cause for the high CRC mortality rate. Understanding the molecular mechanism of CRC liver metastasis could help us to find novel targets for the effective treatment of this deadly disease. Using weighted gene co-expression network analysis on the sequencing data of $\mathrm{CRC}$ with and with metastasis, we identified 5 colorectal cancer liver metastasis related modules which were labeled as brown, blue, grey, yellow and turquoise. In the brown module, which represents the metastatic tumor in the liver, gene ontology (GO) analysis revealed functions including the G-protein coupled receptor protein signaling pathway, epithelial cell differentiation and cell surface receptor linked signal transduction. In the blue module, which represents the primary CRC that has metastasized, GO analysis showed that the genes were mainly enriched in GO terms including G-protein coupled receptor protein signaling pathway, cell surface receptor linked signal transduction, and negative regulation of cell differentiation. In the yellow and turquoise modules, which represent the primary non-metastatic CRC, 13 downregulated CRC liver metastasisrelated candidate miRNAs were identified (e.g. hsa-miR-204, hsa-miR-455, etc.). Furthermore, analyzing the DrugBank database and mining the literature identified 25 and 12 candidate drugs that could potentially block the metastatic processes of the primary tumor and inhibit the progression of metastatic tumors
\end{abstract}

Correspondence to: Professor Zu-Hua Gao, Department of Pathology, The Research Institute of McGill University Health Center, 1001 Boulevard Décarie, Montreal, Québec H4A 3J1, Canada E-mail: zu-hua.gao@mcgill.ca

Key words: weighted gene co-expression network analysis, colorectal cancer liver metastasis, gene module, anti-metastasis drugs in the liver, respectively. Data generated from this study not only furthers our understanding of the genetic alterations that drive the metastatic process, but also guides the development of molecular-targeted therapy of colorectal cancer liver metastasis.

\section{Introduction}

Colorectal cancer (CRC) ranks third in men and second in women among common malignancies (1). Approximately $9 \%$ of cancer-related deaths are caused by CRC (2). An important reason for the high cancer-related mortality rate is that CRC patients are prone to liver metastases. An epidemiological survey showed that $\leq 25 \%$ of patients with CRC developed liver metastases at the time of diagnosis and another $20 \%$ of patients developed liver metastases after resection of the primary tumor (3). Moreover, radical resection of the metastatic lesions in the liver could not be achieved in $80-90 \%$ of patients. In patients with unresectable CRC liver metastases, the 5-year survival rate is close to $0 \%$. Even if the metastases were completely resected, the median survival time is only $\sim 35$ months (4). Thus, it is extremely important to study the molecular mechanisms underlying CRC liver metastases and to find novel targets for the effective treatment of this deadly disease.

During the process of malignant progression, metastasisassociated genetic mutations accumulate in a small fraction of cells within the primary tumor. Once these cells acquire sufficient metastasis-related genetic alterations, they start to undergo a process including local infiltration, lymphovascular invasion, travel within the blood or lymph, extravasation, and angiogenesis at a distant site (5). To unveil the molecular genetic changes in the tumor cell that are responsible for the metastatic process, we sequenced tumor samples from 3 groups of patients: primary CRCs without liver metastasis, primary CRCs with liver metastasis, and the metastatic tumors in the liver. The data have been uploaded into the Gene Expression Omnibus (GEO) database (GSE72718).

Currently, the treatment of CRC liver metastasis is limited to surgery with or without adjuvant chemo-radiation. This 
course of treatment has some limitations. Firstly, not all CRC liver metastases are surgically resectable. Secondly, chemoradiation can cause serious damage to normal tissue such as bone marrow suppression and compromises the patient's overall health (6,7). Thirdly, adjuvant chemo-radiation therapy may directly lead to liver injury and increase complications after liver resection $(8,9)$. In recent years, molecular-targeted therapy has emerged as an effective strategy for the treatment of metastatic cancer (10). Molecular-targeted therapy can be used as a therapeutic means to treat patients with colorectal cancer liver metastasis who are unable to undergo surgical treatment, or patients with unresectable tumors. Molecular-targeted therapy could also act as a new adjuvant therapy after surgical resection to reduce the rate of cancer recurrence. In order for molecular-targeted therapy to be effective, it requires precise identification of all of the potential molecular targets that are responsible for the metastatic process.

Model organisms can not only illustrate local characteristics of gene regulatory networks, but also support comprehensive and systematic analyses of regulatory signaling pathways. Thus, investigation of model organisms has become a desirable method for disease research and drug discovery in recent years $(11,12)$. Weighted gene co-expression network analysis (WGCNA) is a common modular analysis technique that has been used to identify and screen biomarkers or therapeutic targets of different diseases (13). In this study, we used WGCNA to identify colorectal cancer liver metastasis related gene modules. microRNAs (miRNA) regulating these modules were predicted using the miRTarBase database. The functions of the target genes of the miRNAs and the modules were analyzed with the DAVID database. Moreover, using the DrugBank database (14), we identified candidate drugs that can regulate colorectal cancer liver metastasis related modules. Our study uncovered some new targets and candidate antimetastasis drugs for future research into the mechanism and molecular-targeted therapy of CRC liver metastasis.

\section{Materials and methods}

Sample collection and gene sequencing. With the approval of the institutional ethics review board, we collected primary non-metastatic colorectal tumor (PNMCT) samples from 10 patients with colorectal cancer who had no liver metastases within ten years of follow-up, primary metastatic colorectal tumor (PMCT) samples and their paired metastatic CRC samples in the liver (LMCT) from 9 patients with CRC who had liver metastases (Fig. 1A) We used the next generation sequencing technology of Affymetrix (Human Transcriptome Array 2.0) to detect gene expression in the 28 samples and then uploaded the data to the GEO database (GEO no. GSE72718). The technology platform, samples and groups used in this study are shown as Table I. Based on the sequencing results, we established a heatmap of differentially expressed genes (DEGs) in this study (Fig. 1B).

Weighted gene co-expression network analysis (WGCNA). WGCNA, a common modular analysis technique, has been used to identify and screen biomarkers or drug targets for complex diseases. We used the genefilter package of the programming language and software environment $\mathrm{R}$ to filter out genes with smaller differences in expression between the three groups than within the groups prior to WGCNA. First, we used WGCNA to construct the correlation matrix of co-expressed genes. Elements in the co-expression matrix included pairwise correlation coefficients between genes (i.e., the correlation coefficient between each gene pair $\mathrm{m}$ and $\mathrm{n}$ was: $\mathrm{Smn}=|\operatorname{cor}(\mathrm{m}, \mathrm{n})|$; thus, the co-expression matrix was: $\mathrm{S}=[\mathrm{Smn}])$. Next, the power adjacency function amn $=$ power $(\mathrm{Smn}, \beta)=\mathrm{ISmnl} \beta$ was used as an indicator to measure the relationships between genes. According to the principle of scale-free networks, the weighting coefficient $\beta$ was determined, and the matrix $S$ was converted into an adjacency matrix $A=[a m n]$. Then, a hierarchical clustering tree was constructed with different branches of the tree representing different gene modules. After module identification, the p-value from the significance test for the expression of each gene between different groups was calculated using the t-test based on the phenotypic data of the groups. Gene significance was indicated using the $\log \mathrm{p}$-value. The significance of each module was defined as the mean value of gene significance for genes within the module. Modules with an increased significance might have a correlation with the presence of a specific disease. Modules were clustered in meta-modules for the module eigengene dendrogram, which is a dendrogram of all differentially expressed probesets clustered based on a dissimilarity measure. Each line of the dendrogram corresponds to a probeset. The multi-colored bar below the dendrogram shows the modules identified using the dynamic cutting method, with each gene color-coded based on its module assignment. Module gene members are not always adjacent to each other because WGCNA modules do not comprise only leaves with their direct ancestors (Fig. 1C).

Gene ontology (GO) analysis. To understand the function of modules and target genes, we used The Database for Annotation, Visualization and Integrated Discovery (DAVID) v6.7 to identify the differentially expressed (DE) GO terms of DEGs and target genes of DE microRNA in modules. Fisher's exact test was used to identify the significant DE GO term. The p-value cut-off is 0.05 .

Establishing miRNA-target gene network. We identified the modules that were gradually downregulated during colorectal liver metastasis progression (from PNMCT through PMCT to LMCT). Based on the relationship between miRNA and the target gene, gradually downregulating the miRNAs in the modules might gradually increase target gene expression and thus contribute to the progression and metastasis of CRC. We used the miRTarBase database to predict the confirmed target genes of miRNAs. Cytoscape software was used to establish the miRNA-target gene network.

Establishing candidate drug-module network. The DrugBank database is a unique bioinformatics and cheminformatics resource that combines detailed drug data with comprehensive drug target information (14). The database contains 8,198 drug entries including 1,985 FDA-approved small molecule drugs, 204 FDA-approved biotech (protein/peptide) drugs, 93 nutraceuticals and over 6,000 experimental drugs. In this study, 
Table I. Summary of data series.

\begin{tabular}{llll}
\hline Series & Array platform & \multicolumn{1}{c}{ Samples } & Experiment design \\
\hline GSE72718 & GPL17586.0 & GSM1868934-GS & Liver metastatic \\
& [HTA-2_0] & M1868942 & colorectal tumor \\
& Affymetrix Human & GSM1868943-GS & Primary metastatic \\
& Transcriptome & M1868951 & colorectal tumor \\
& Array 2 & GSM1868952-GS & Primary non-metastatic \\
& & M1868961 & colorectal tumor \\
\hline
\end{tabular}

A

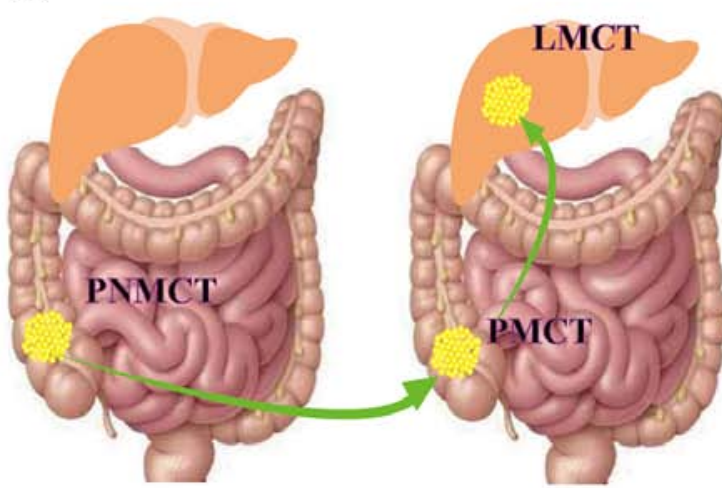

B

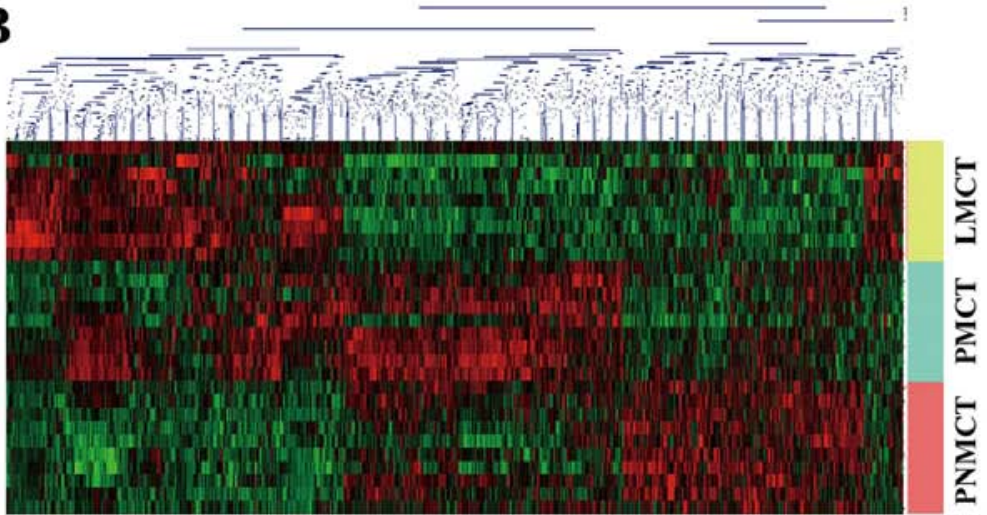

C

Cluster Dendrogram

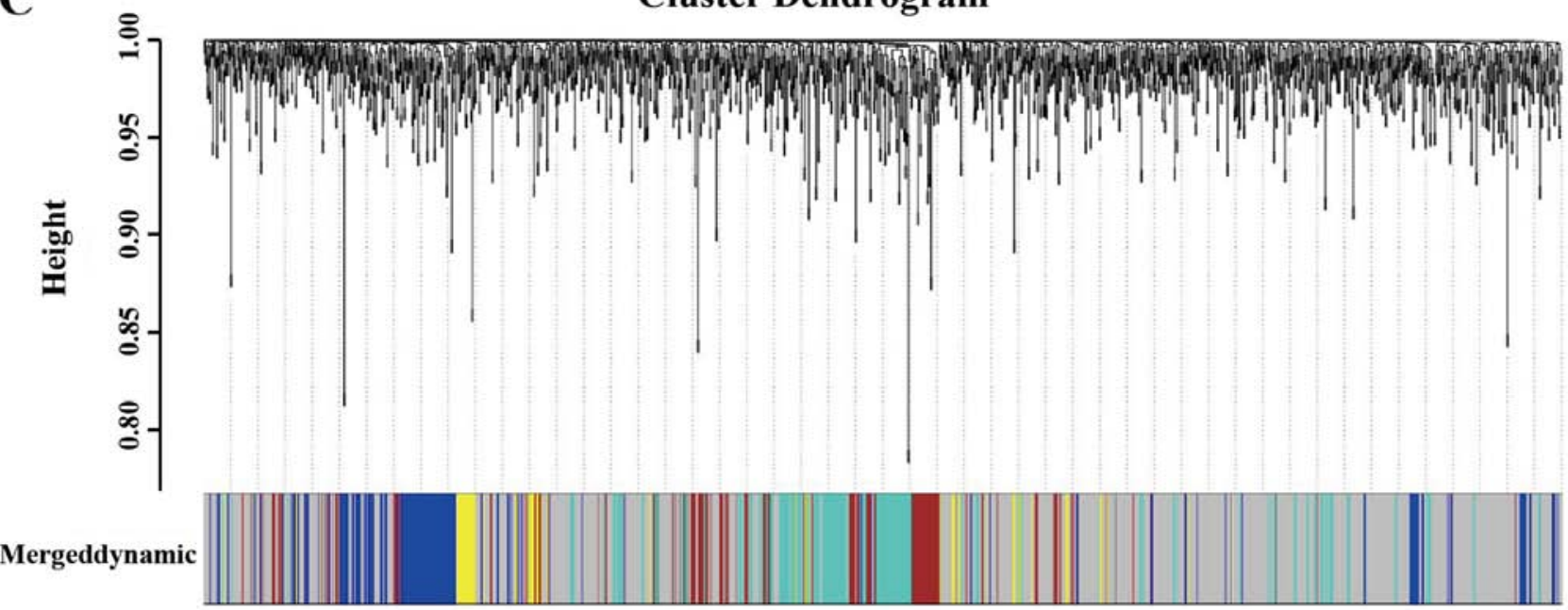

Figure 1. WGCNA of the colorectal cancer liver metastasis genome sequencing data. (A) Illustration of the 3 groups of tumor specimens that have been sequenced. LMCT, metastatic tumor in the liver; PNMCT, primary non-metastatic colorectal cancer; PMCT, primary metastatic colorectal cancer. (B) Based on the genome sequencing results, we established the heatmap of DEGs. (C) Modules were clustered in meta-modules for the module eigengene dendrogram, a dendrogram of all differentially expressed probesets clustered based on a dissimilarity measure. Each line of the dendrogram corresponds to a probeset. The multi-colored bar below the dendrogram shows the modules identified using the dynamic cutting method with each gene color-coded based on module assignment.

we used the DrugBank database Version 4.3 (http://www. drugbank.ca/) to identify the candidate drugs that target the genes in our modules. Finally, Cytoscape software was used to establish the candidate drug-module gene network.

Literature mining on candidate drugs that connect regulatory network modules. To identify novel antitumor metastasis drugs, we analyzed 119 candidate drugs related to the modules.
Perl was used to write a program for literature mining. ActivePerl 5.16.2 was used to mine literature information from PubMed (NCBI). The mining scope was set to include titles and abstracts, along with candidate drug names, modular gene names and 'cancer' as key words. This search provided reports related to each candidate drug and cancer. Integrating the candidate drug screening results, literature mining results and the modular genes, we identified the drugs with the greatest 
Brown module

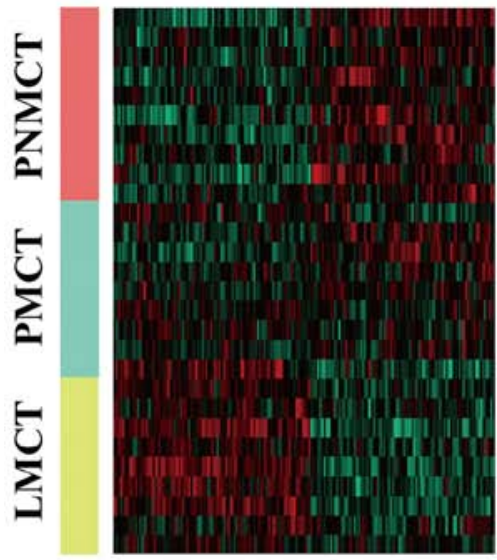

Blue module

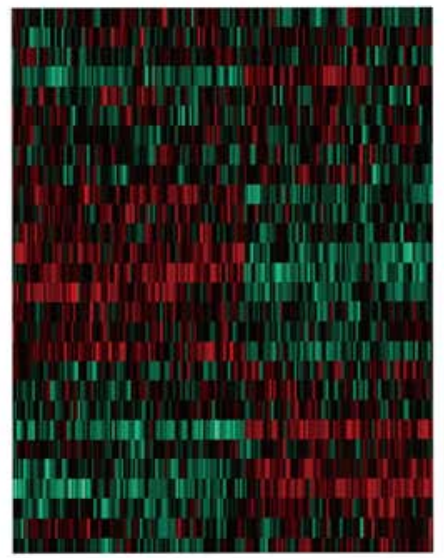

Grey module

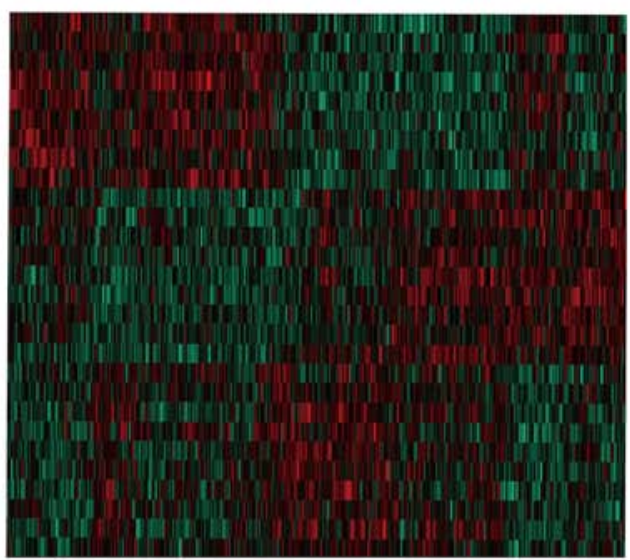

Turquoise module

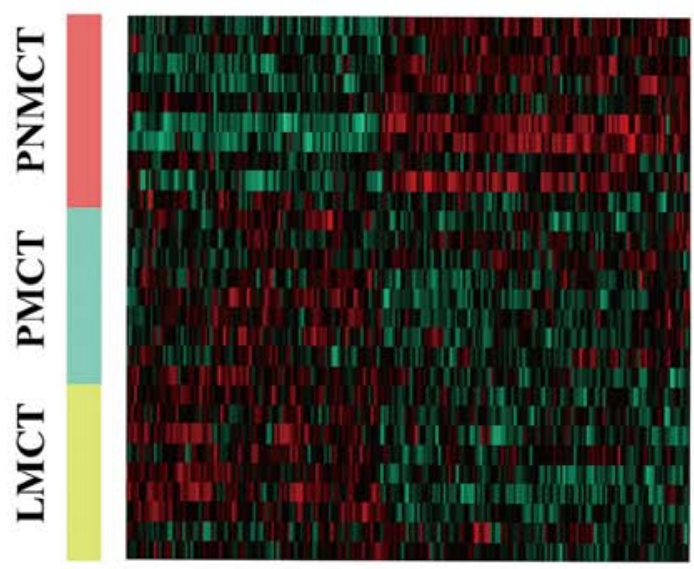

Yellow module

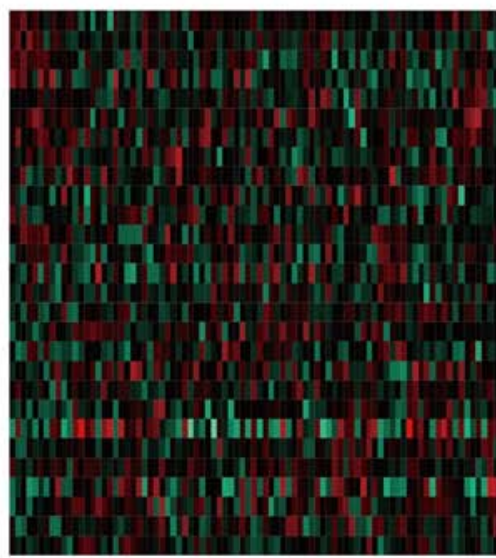

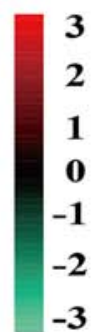

Figure 2. Heat maps of WGCNA modules. WGCNA of the sequencing data identified five colorectal cancer liver metastasis related modules (i.e., brown, blue, grey, turquoise and yellow).

potential antitumor activity for the modular core function groups.

Statistical analysis. The t-test was used to identify the DEGs and the DE modules based on WGCNA. Fisher's exact test was used to identify the significant GO terms. The p-value cut-off is 0.05 .

\section{Results}

Screening the gene modules. Using WGCNA to analyze the sequencing data, we found a total of 5 functional modules for colorectal cancer liver metastasis (i.e., brown, blue, turquoise, grey and yellow module) (Fig. 2). Modules with a significant increase in gene expression might have a correlation with the presence of a specific disease. Based on the p-value from the significance test for each module, the brown module and blue module were significantly associated with LMCT and PMCT, respectively. The grey module, turquoise module and yellow module were significantly associated with PNMCT $(\mathrm{p}<0.05)$ (Fig. 3).

miRNA-mRNA regulatory network. In addition to protein coding genes, there were some DE miRNAs in the five modules. As shown in Figs. 2 and 3, the expression of the miRNAs was gradually downregulated in the turquoise and yellow modules. According to the principle of targeted gene suppression by miRNAs, the inhibitory effect of the downregulated miRNA on the target gene was reduced, resulting in high expression of the target gene. Therefore, the downregulated miRNA in the yellow and turquoise modules may play an important role on the development of CRC. There were a total of two miRNAs in the yellow module (i.e., hsa-miR-3151 and hsa-miR-4518). There were a total of 11 miRNAs in the turquoise module (i.e., hsa-miR-320d; hsa-miR-4690; hsa-miR-455; hsa-miR-4270; hsa-miR-892c; hsa-miR-378a; hsa-miR-4740; hsa-miR-204; hsa-miR-4417; hsa-miR-539; hsa-miR-3158). We used the miRTarbase database to predict the confirmed target genes of miRNAs. The results showed that there were 256 target genes of the two miRNAs in the yellow module (Fig. 4) and 2,502 target genes of 11 miRNAs in the turquoise module (Fig. 5).

Functional analyses of the gene modules and target genes. To understand the function of the module and the target genes of the miRNAs, the DAVID database was used to analyze their GO terms. The results showed that the main functions of the blue module were $5 \mathrm{GO}$ terms including G-protein coupled 


\begin{tabular}{|c|c|c|c|}
\hline $\begin{array}{c}0.73 \\
1.01 \mathrm{e}-05\end{array}$ & $\begin{array}{c}-0.04 \\
8.43 e-01\end{array}$ & $\begin{array}{c}-0.67 \\
8.42 \mathrm{e}-05\end{array}$ & Brown \\
\hline $\begin{array}{c}0-.47 \\
1.09 \mathrm{e}-02\end{array}$ & $\begin{array}{c}-0.30 \\
1.23 \mathrm{e}-01\end{array}$ & $\begin{array}{c}0.75 \\
3.87 \mathrm{e}-06\end{array}$ & Turquoise \\
\hline $\begin{array}{c}-0.59 \\
9.73 e-04\end{array}$ & $\begin{array}{c}0.61 \\
5.88 \mathrm{e}-04\end{array}$ & $\begin{array}{c}-0.02 \\
9.23 \mathrm{e}-01\end{array}$ & Blue \\
\hline $\begin{array}{c}-0.69 \\
4.19 e-05\end{array}$ & $\begin{array}{c}0.18 \\
3.50 \mathrm{e}-01\end{array}$ & $\begin{array}{c}0.50 \\
7.05 \mathrm{e}-03\end{array}$ & Yellow \\
\hline $\begin{array}{c}-0.38 \\
4.42 \mathrm{e}-02\end{array}$ & $\begin{array}{c}-0.47 \\
1.20 \mathrm{e}-02\end{array}$ & $\begin{array}{c}0.83 \\
4.78 \mathrm{e}-08\end{array}$ & Grey \\
\hline LMCT & PMCT & PNMCT & \\
\hline
\end{tabular}

Figure 3. The statistical test of DE modules based on WGCNA. Based on the p-value of each module, the brown and blue modules were significantly associated with LMCT and PMCT, respectively. The grey, turquoise and yellow modules were significantly associated with PNMCT $(\mathrm{p}<0.05)$.

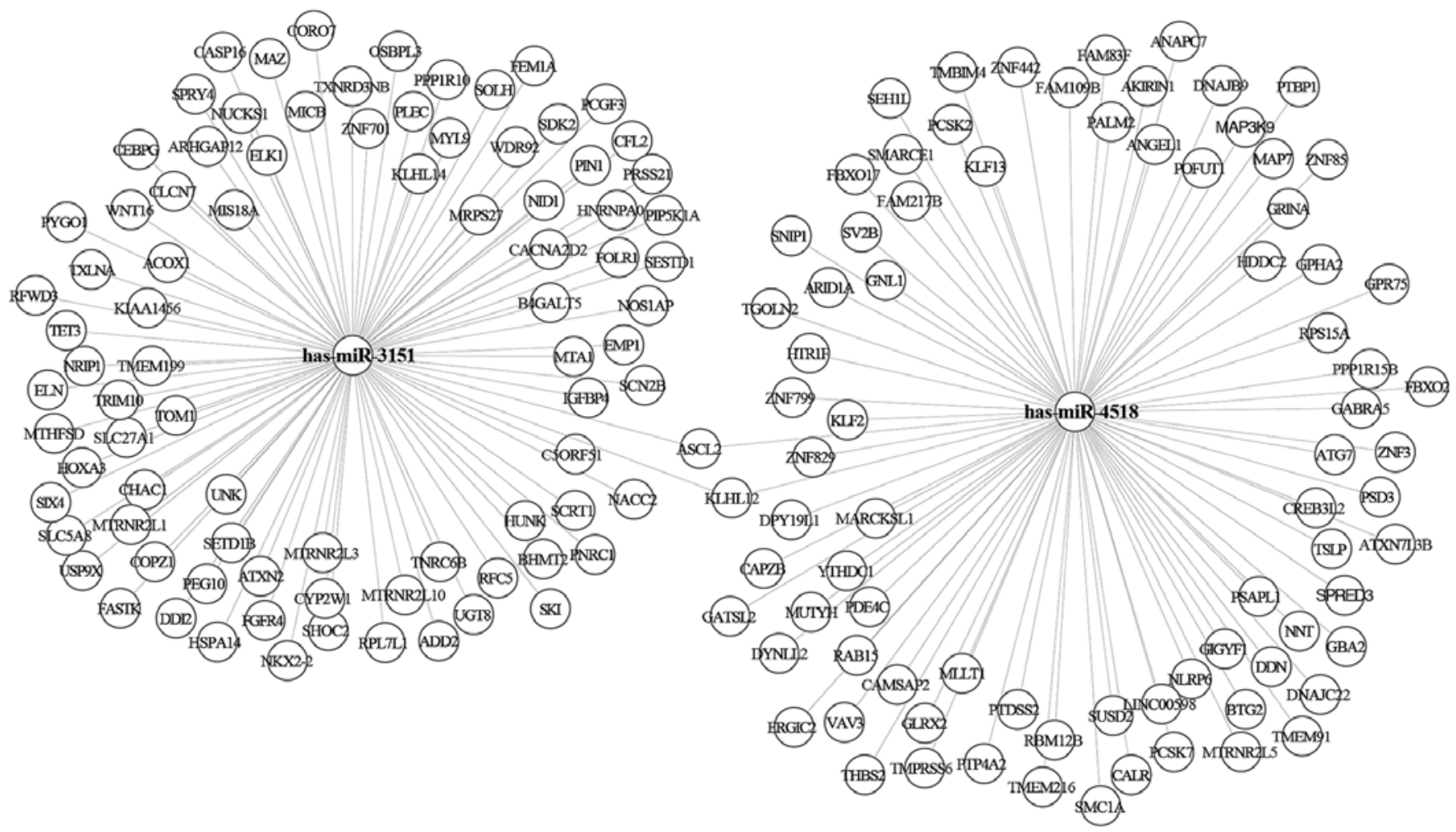

Figure 4. The miRNA-target mRNA regulatory network of the yellow module. According to the principle of targeted gene suppression by miRNAs, downregulation of the miRNA will reduce its inhibitory effect on the target gene and consequently result in high expression of the target gene. Therefore, the downregulated miRNAs in the yellow module may play an important role on the development of CRC. We predicted the target mRNAs of DE miRNAs and established the miRNA-target mRNA network of the yellow module.

receptor protein signaling pathway, cell surface receptor linked signal transduction, and negative regulation of cell differentiation. The GO terms G-protein coupled receptor protein signaling pathway, epithelial cell differentiation and cell surface receptor linked signal transduction were included in the main functions of the brown module. The functions of the miRNA target genes in the turquoise module included cell motion, wound healing, and regulation of cell adhesion. There were 4 main GO terms for the miRNA target genes in the yellow module (e.g., regulation of cellular protein metabolic 

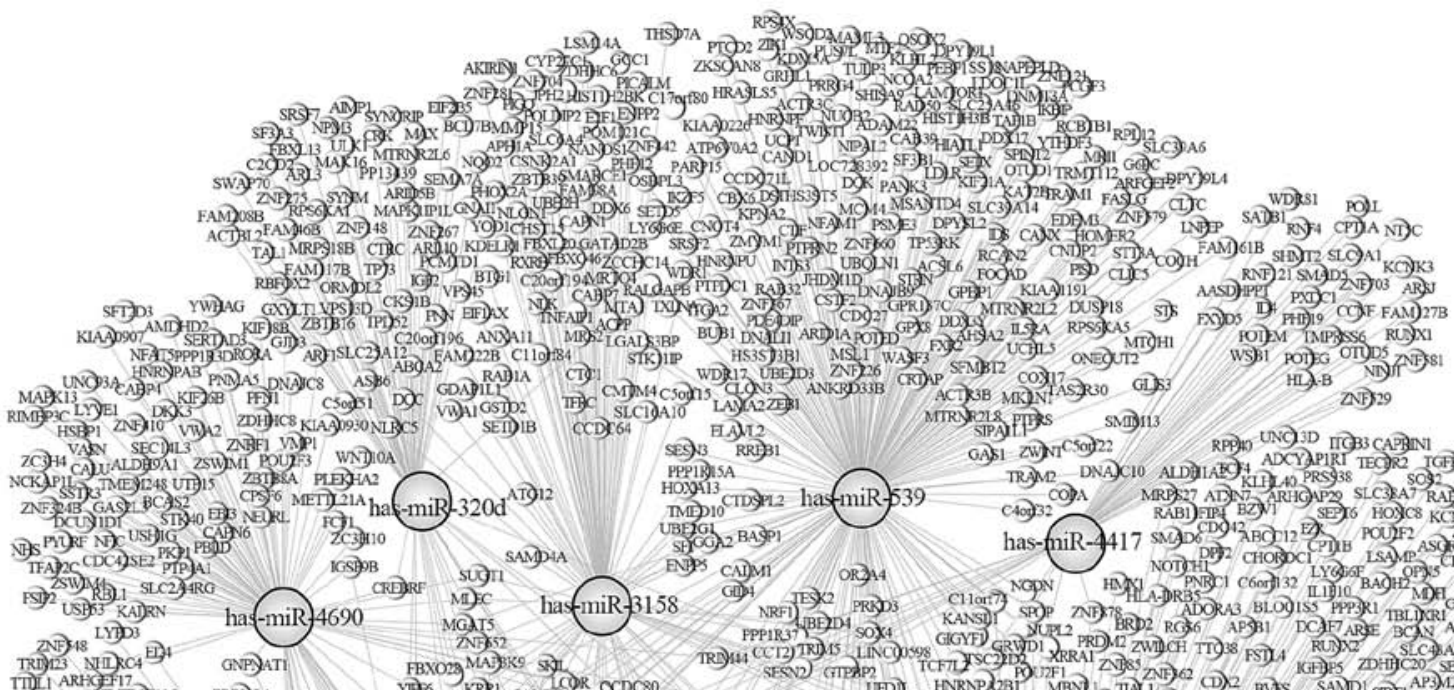

OASI ZWMNCSOID2

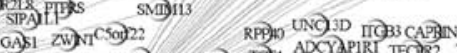

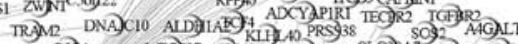

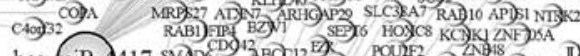

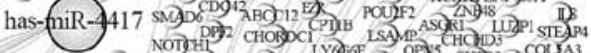

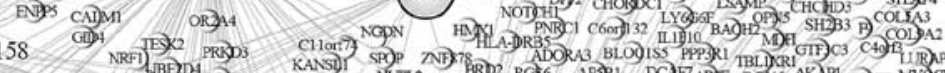

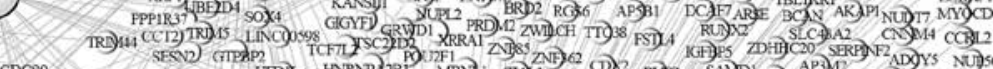

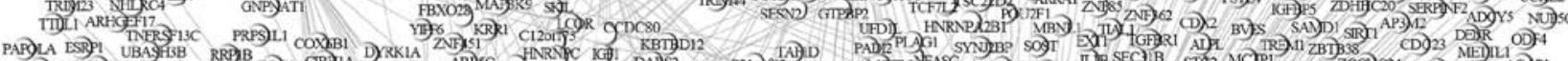

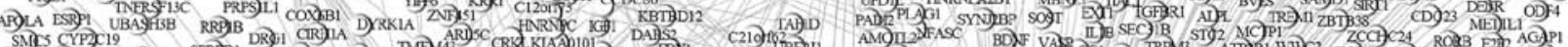

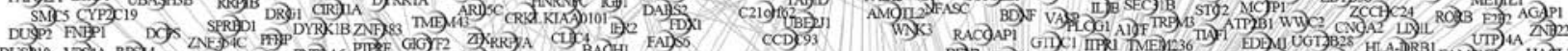

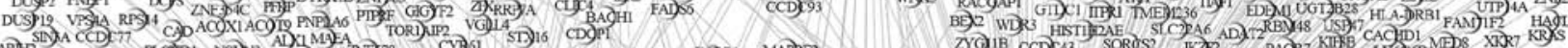

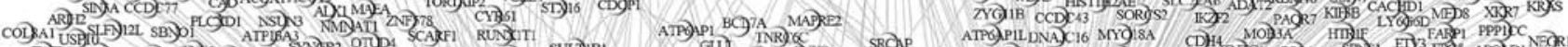

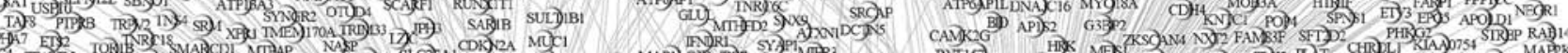

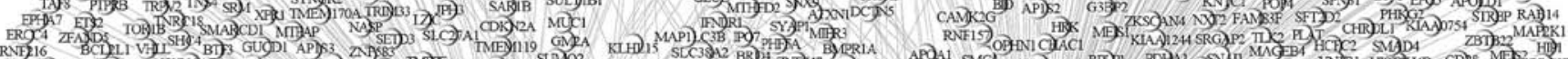

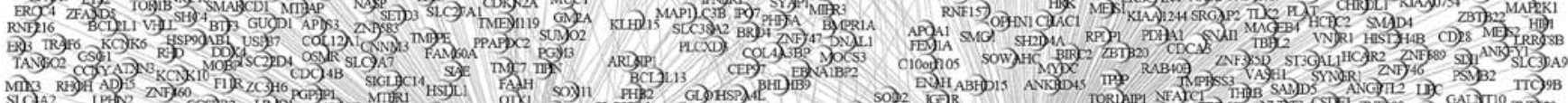

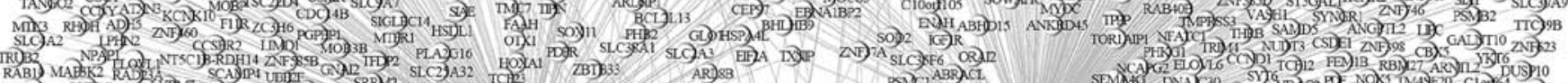

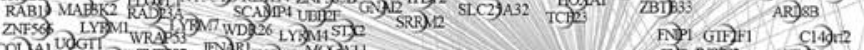
(OI) A U. UTI WRA KATX AYYCCD 38 RASSE2

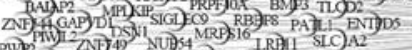

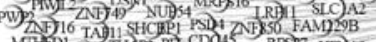

Figs DEA PABDCLIJP RP

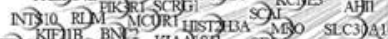
EFA AIF

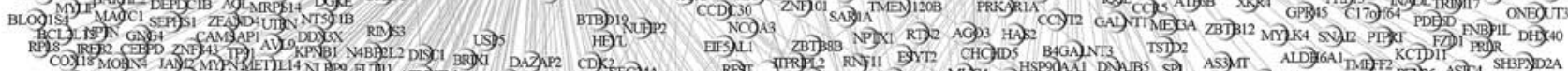

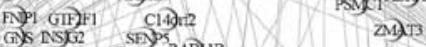

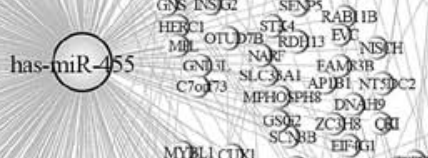

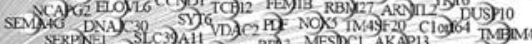

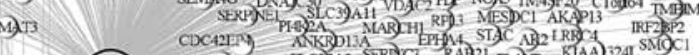

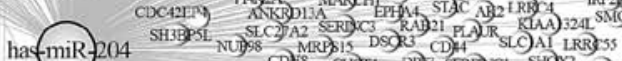

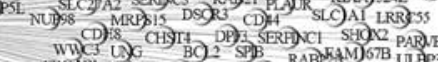

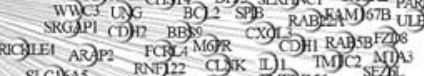

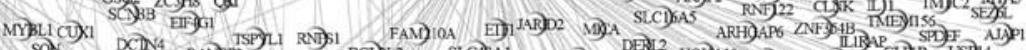

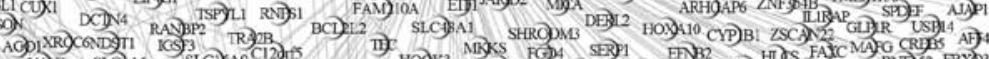

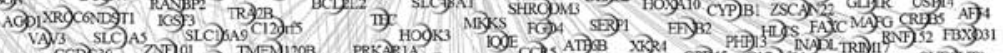

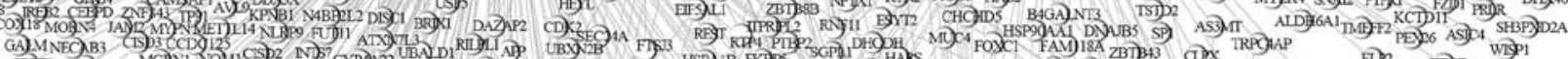

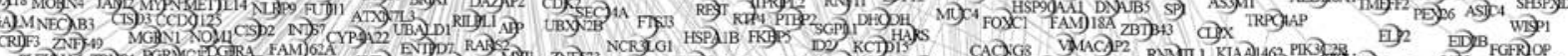

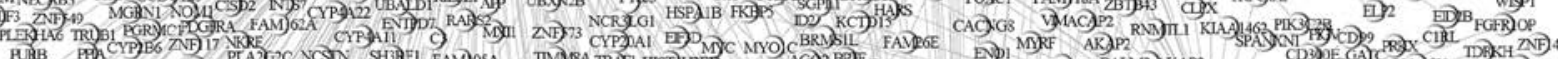

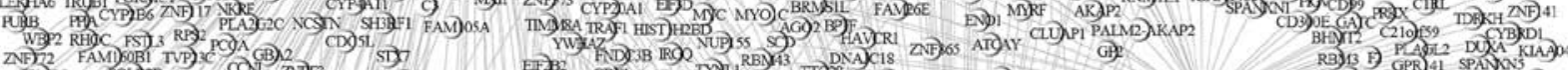

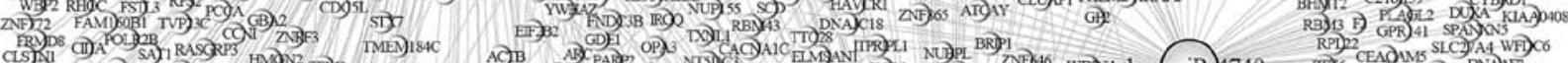

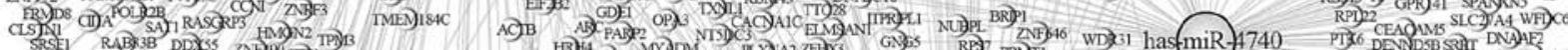

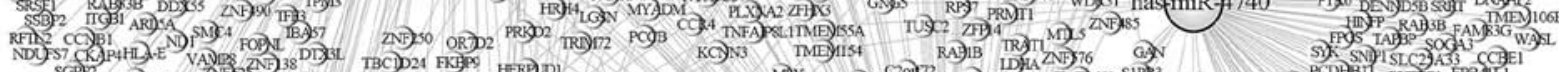

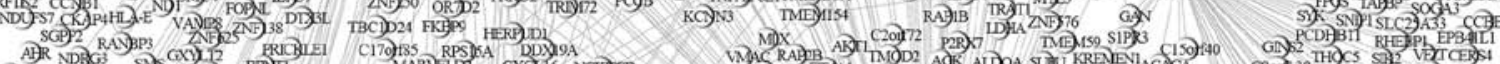

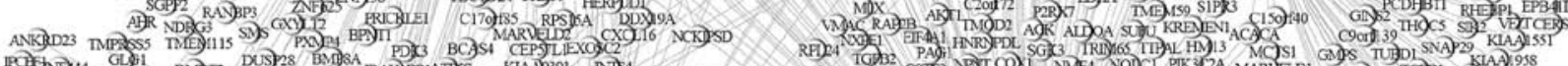

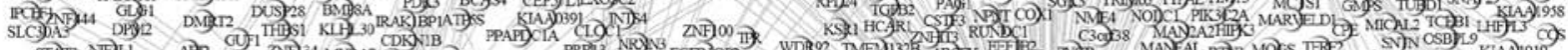
$\begin{array}{llll} & \end{array}$

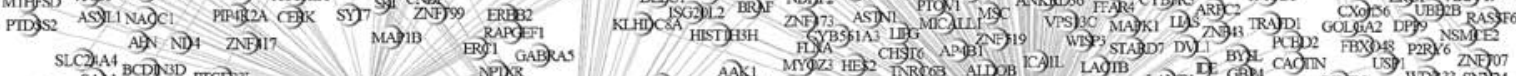

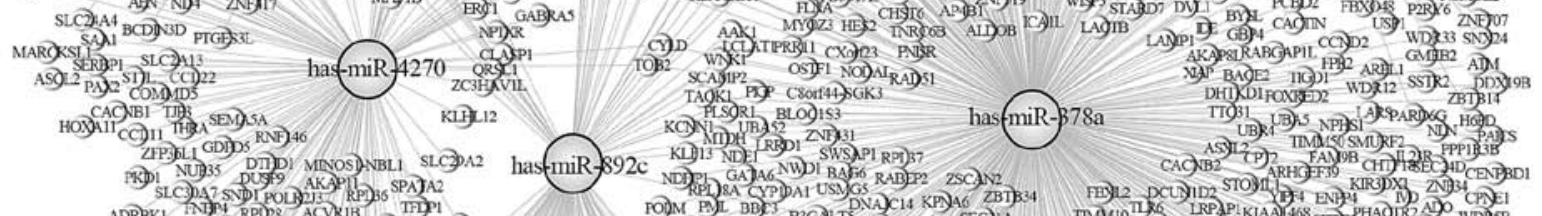

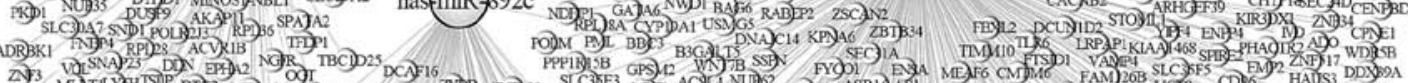

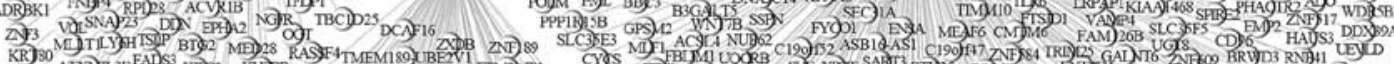

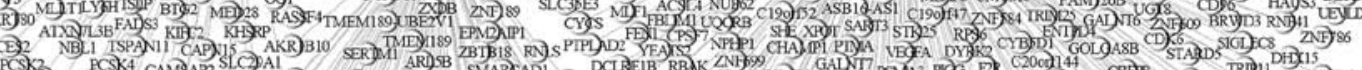

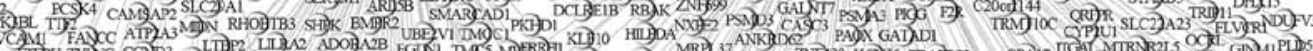

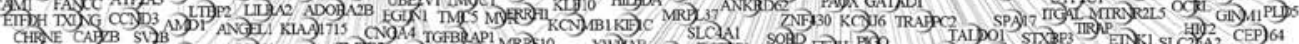
CABVB SUBB

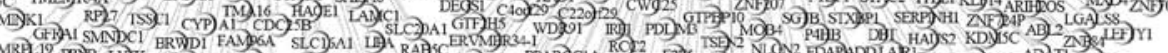

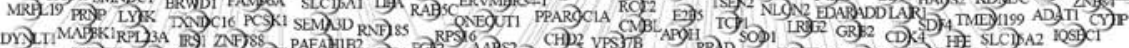

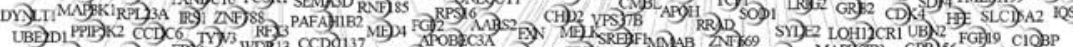

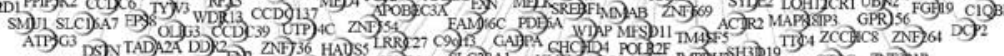

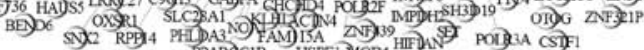

Figure 5. The miRNA-target mRNA regulatory network of the turquoise module. Consistent with the method used in Fig. 4 , we predicted the target mRNAs of DE miRNAs and established the miRNA-target mRNA network of the turquoise module. 
Blue module

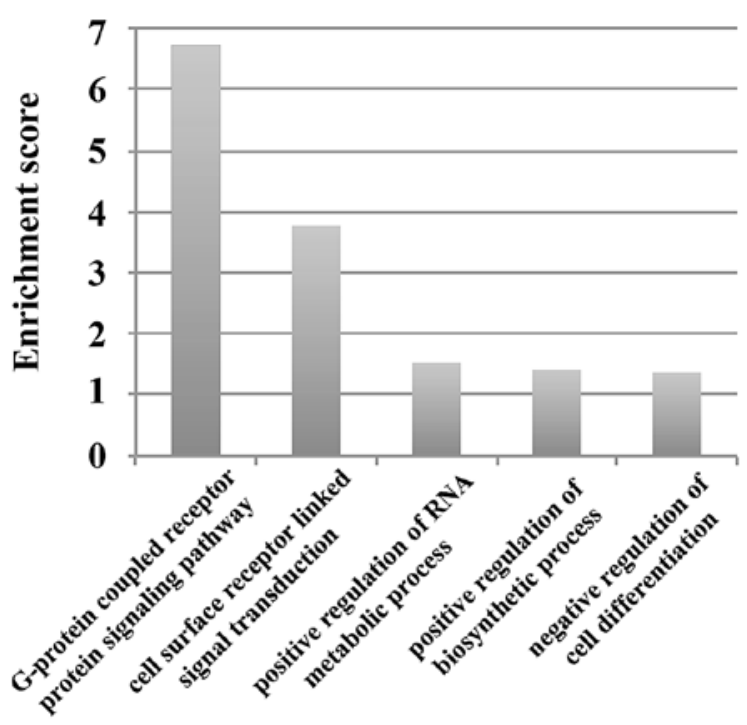

Turqouise module

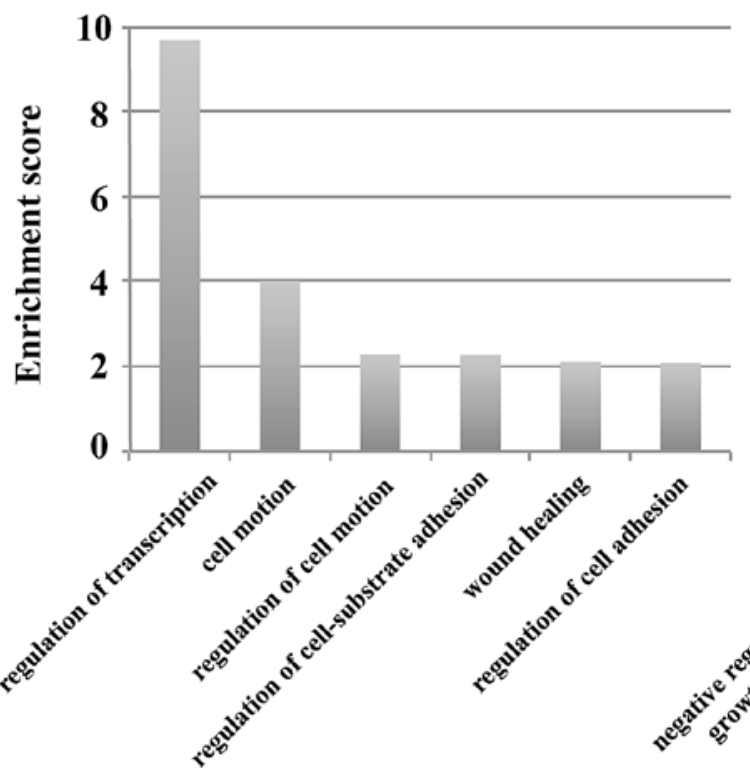

Brown module

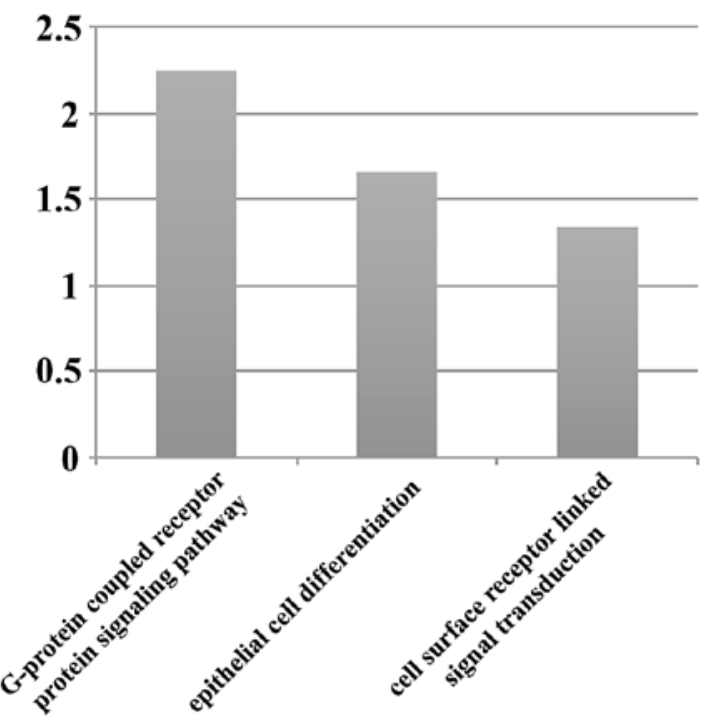

Yellow module

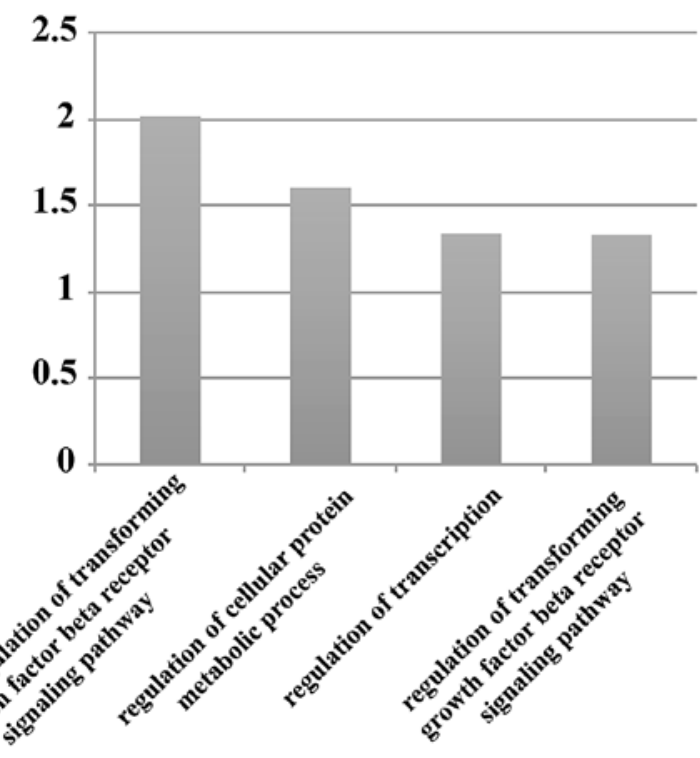

Figure 6. Functional analysis of DEGs (in the blue and brown modules) and the target genes of DE miRNAs (in the turquoise and yellow modules). The DAVID database was used to analyze the function of colorectal cancer liver metastasis related modules. Enrichment $s c o r e=-\log p$-value, $\mathrm{p}<0.05$.

process, and regulation of transcription). Enrichment score $=-$ $\log$ p-value, $\mathrm{p}<0.05$ (Fig. 6).

Identification of candidate drugs that regulate the modules. We found that the genes in the brown and blue modules were gradually upregulated from PNMCT to LMCT; therefore, the two modules may be closely related to the progression of CRC. In order to identify potential new therapies for colorectal cancer liver metastasis, we used the DrugBank database to isolate the candidate drugs that regulate these two modules. The DrugBank database is a unique bioinformatics and cheminformatics resource that combines detailed drug data with comprehensive drug target information. Based on the DrugBank database, we identified 83 candidate drugs as regulators of the brown module. Among the DEGs in the brown module, adrenocepor 1D (ADRA1D), 5-hydroxytryptamine receptor 1D (HTR1D), lysyl-tRNA synthetase (KARS), lipocalin 9 (LCN9) and methionine sulfoxide reductase A (MSRA) were the important targets of drug regulation. A total of 43 candidate drugs were identified as regulators of the blue module. Among the DEGs in the blue module, calcitonin related polypeptide $\beta$ (CALCB), formyl peptide receptor 1 (FPR1), 5-hydroxytryptamine receptor 2B (HTR2B), recoverin (RCVRN) and retinoid $\mathrm{x}$ receptor (RXR) were the important targets of drug regulation. Cytoscape software was used to establish the candidate drug-module network (Fig. 7). Of note, there were 17 candidate drugs (for example, Ropinirole, Dihydroergotamine, Olanzapine, Pramipexole and Doxepin) that can regulate the two modules among these candidate drugs. 


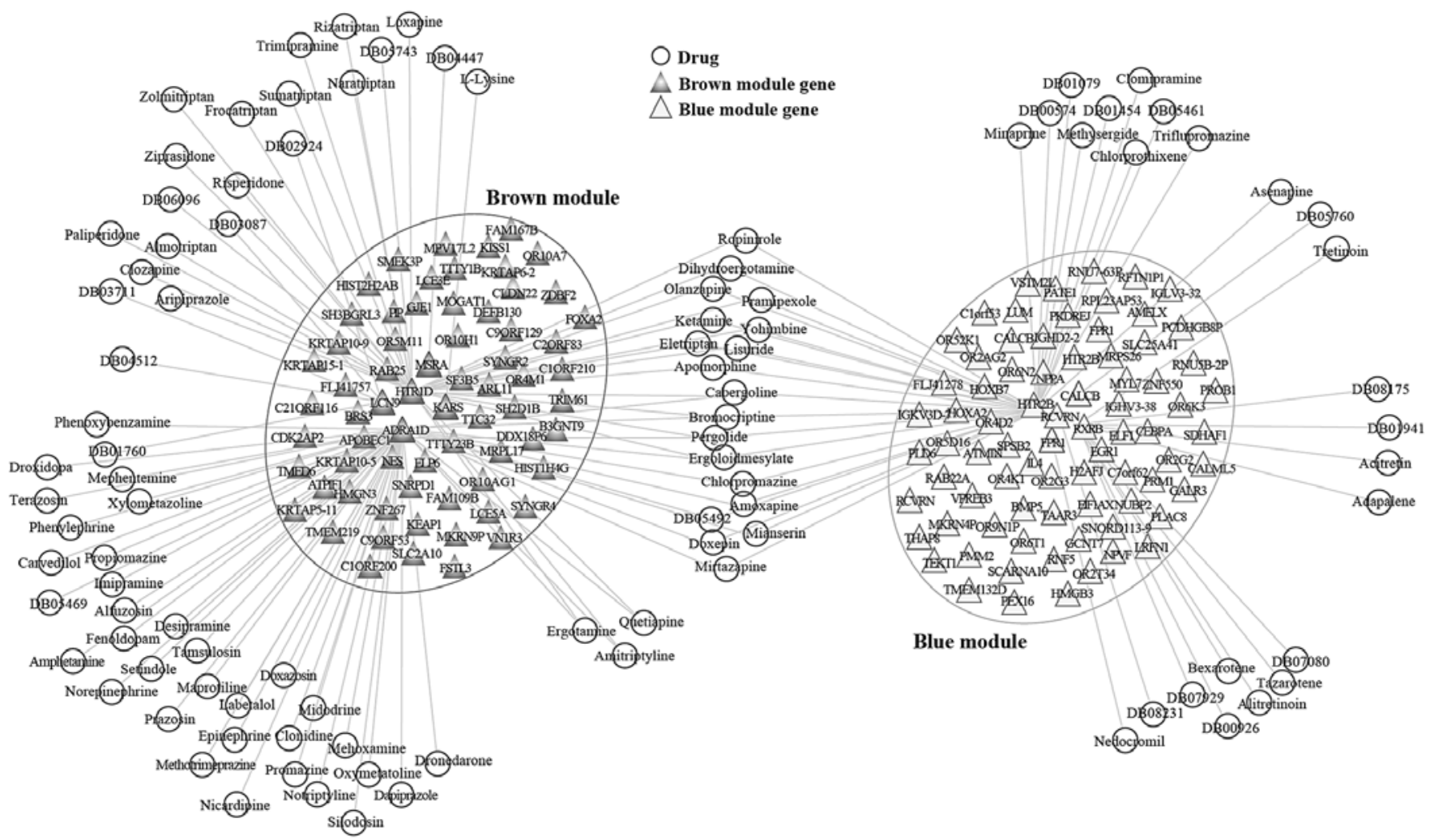

Figure 7. The candidate drug-module network. The DrugBank database was used to identify the candidate drugs that regulate the brown and blue modules. Cytoscape software was used to establish the candidate drug-module network. Eighty-three and 43 candidate drugs were identified as regulators of the brown and blue modules, respectively. Among these drugs, 17 drugs can regulate both modules.

Screening of potential new anti-metastasis drugs. To search for novel anti-metastasis drugs, we conducted literature mining of the cancer-related research on the 83 candidate drugs that regulate the brown module and the 43 candidate drugs that regulate the blue module. The number of reports was an indicator of the extent of research on the drug's use in cancer treatment. In particular, fewer reports were associated with a higher likelihood that the drug could become a novel antimetastasis drug. The results revealed that 24 candidate drugs have been studied extensively and have already been proven to be effective anticancer drugs among the candidate drugs that regulate the brown module. These drugs included Epinephrine, Ketamine, Prazosin, Labetalol (the number of relevant reports was $>100$ ). Twenty-five candidate drugs, however, have not been extensively studied with respect to their anticancer value, such as Droxidopa, Eletriptan, Loxapine (the number of relevant reports was $\leq 10$ ). Among them, 9 candidate drugs, such as Almotriptan, Dapiprazole, DB03711 (NXN-188), have never been studied with respect to cancer (Fig. 8A). The 25 candidate drugs might have the potential to suppress CRC liver metastases through their regulation of the genes in the brown module.

Among the drugs that regulate the blue module, 14 candidate drugs have been studied extensively such as Tretinoin, Bromocriptine, Chlorpromazine. Twelve candidate drugs have not been extensively studied with respect to their anticancer value (the number of relevant reports was $\leq 10$ ). Among them, 3 candidate drugs Minaprine, DB05461 (OPC-28326), DB05492 (Epicept NP-1), have never been studied with respect to cancer (Fig. 8B). The 12 candidate drugs might have the potential to prevent primary CRC from developing into liver metastases through regulation of the blue module.

\section{Discussion}

Tumor metastasis is a multi-step, multi-stage, and complex process involving multiple pathways and multiple genetic changes. Therefore, research on the genetic alterations associated with the metastatic process is essential for understanding its molecular mechanisms. Next-generation sequencing offers unprecedented tools to unveil the genetic changes underlying CRC liver metastasis.

Tumor metastasis models normally start with mutations of metastasis-related genes in a small fraction of tumor cells. When these genetic changes accumulate to a certain degree, these cells will gradually or randomly acquire a metastatic capacity and then undergo the series of processes which eventually result in distant tumor metastasis (5). On the other hand, some tumors only invade locally without the capacity for distant metastasis. Among our study groups, the tumors that did not metastasize over 10 years of follow-up after the diagnosis of CRC (PNMCT) were not supposed to bear any metastasis-related genes, and thus could serve as a negative control. The metastatic tumors within the liver (LMCT) should theoretically contain all the genetic alterations required for the metastatic process. The group of paired primary CRC samples that have metastasized (PMCT) is supposed to bear a spectrum of metastasis-related genes. This simple group set-up allowed 

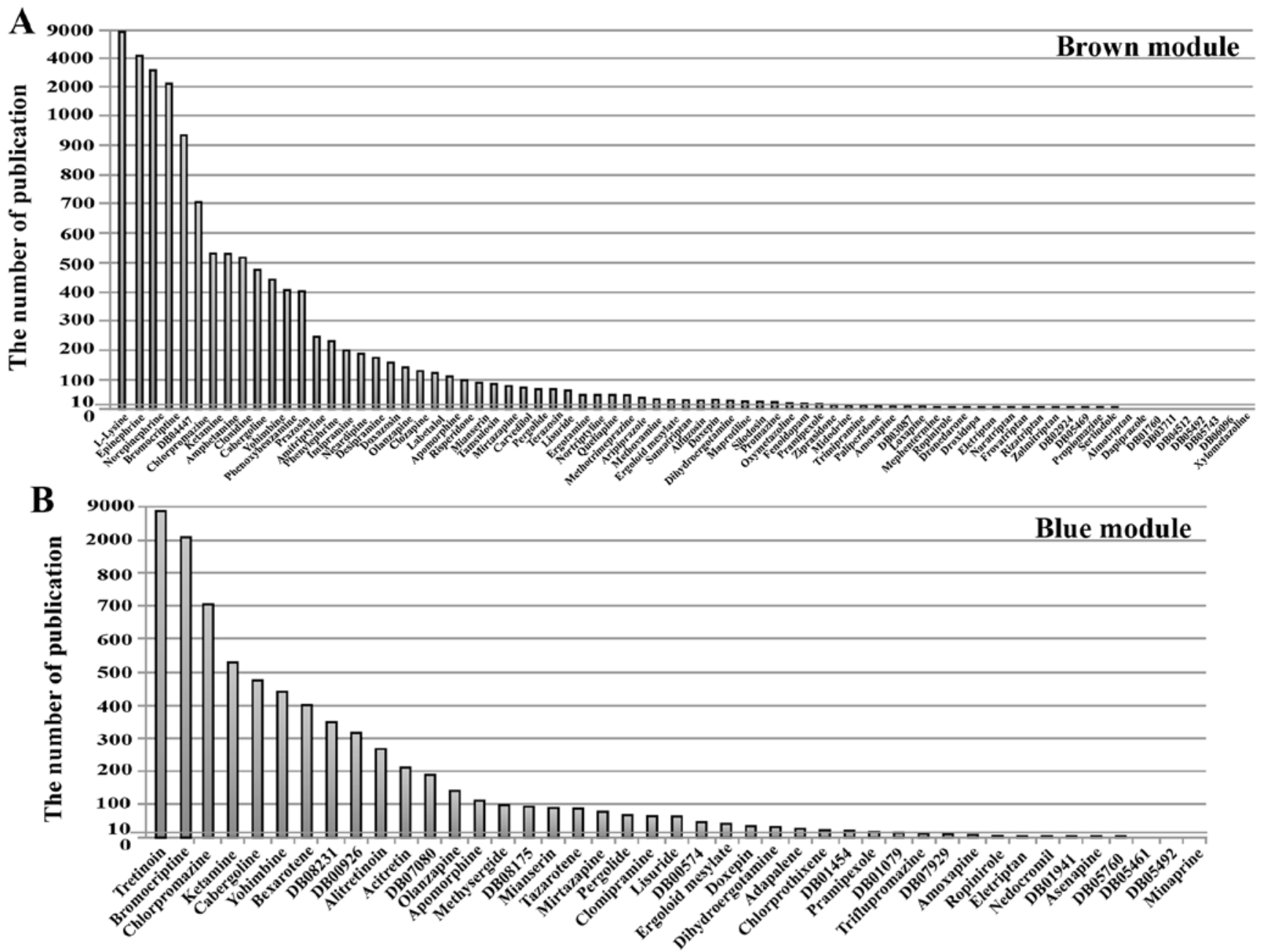

Figure 8. Potential new anti-metastasis drugs that could regulate the DEGs in the brown and blue modules. To identify novel anti-metastasis drugs, we conducted a literature review of the cancer-related research on the 83 candidate drugs that regulate the brown module (A) and the 43 candidate drugs that regulate the blue module (B). The number of reports was an indicator of the extent of research on the candidate drugs use in cancer treatment. In particular, fewer reports were associated with a higher likelihood that the candidate drug could become a new anti-metastasis drug. Twenty-five candidate drugs have the potential to treat colorectal cancer liver metastasis through regulating the genes in the brown module (A). Twelve candidate drugs have the potential to prevent primary CRC from developing into liver metastasis through regulating the blue module (B) the number of relevant reports was $\leq 10$.

us to dynamically and comprehensively explore the genetic changes that occurred during CRC liver metastases.

Studies have shown that genes with expression similarities often exist in samples from different diseases. When these genes are co-expressed, they are often closely associated with disease phenotypes. Thus, gene co-expression network analysis is an important tool to identify these genes (15). Among the five modules screened out by WGCNA in our study, the brown module was closely associated with the phased changes in CRC liver metastasis (Fig. 2). The expression of genes in this module gradually increased during the transition from non-metastasis to metastasis (from PNMCT to LMCT), suggesting that the brown module was positively correlated with the tumor metastatic process. The result of GO analysis showed that the GO terms G-protein coupled receptor protein signaling pathway and cell surface receptor linked signal transduction were the main functions of the brown module. Research indicates that G protein-coupled receptors (GPCRs) have a close relationship with tumor metastasis. Tang et al, found that GPR116, an adhesion G-protein-coupled receptor, can promote breast cancer metastasis (16). Yan et al found G protein-coupled receptor 87 (GPR87) promotes the growth and metastasis of $\mathrm{CD}_{133^{+}}$cancer stem-like cells in hepatocellular carcinoma (17). GPCRs can facilitate tumor metastasis by activating Rho GTPases and cytoskeletal changes. Additionally, GPCRs can supplement nutrition for tumor angiogenesis and thereby provide a path for tumor metastasis. The role of GPCRs in colorectal cancer liver metastasis is presently unknown. Our results suggest that GPCRs have great potential as therapeutic targets for colorectal cancer liver metastasis. Moreover, cell surface receptors play important roles in tumor metastasis. Kim et al found that the cell-surface receptor for complement component $\mathrm{Clq}(\mathrm{gClqR})$ was a key regulator for lamellipodia formation and cancer metastasis (18). Mutation or inactivation of genes encoding cell surface receptors leads to decreased cell adhesion and thereby promotes tumor metastasis, which is an important step underlying this process. Therefore, the brown module that was primarily associated with the GO term function cell surface receptor-linked signal transduction might be critical for CRC liver metastasis. Furthermore, highly 
expressed genes of the blue module were closely associated with the transitional phase of colorectal cancer liver metastasis (PMCT). The GO analysis revealed that the functions of the blue module were consistent with the functions of the brown module. This result indicates that GPCRs and cell surface receptors may participate in the entire colorectal cancer liver metastatic process.

Both the brown and blue modules can be used as targets for anti-metastasis drugs. Drugs directly acting on the brown module can inhibit the progression of the metastatic tumor, whereas drugs acting on the blue module can block or interfere with the process of tumor metastasis, thereby reducing the rate of incidence of CRC liver metastasis. We screened drugs regulating the brown module and blue module using the DrugBank database (Fig. 7). A total of 83 and 43 medicinal monomers regulated the brown and blue modules, respectively. Notably, 19 medicinal monomers could simultaneously regulate both modules, among which olanzapine, ketamine, yohimbine, and chlorpromazine have been extensively used in anticancer therapy. Guo et al found that olanzapine induced autophagy to inhibit glioma stem-like cells proliferation (19). Recent research has indicated that the antipsychotic chlorpromazine can inhibit sirtuin 1 to treat CRC (20). Among the drugs targeting the brown module, bromocriptine reduces the risk of breast cancer brain metastasis, and cabergoline treatment of metastatic breast cancer has entered the clinical trial stage $(21,22)$. Additionally, among the drugs targeting the blue module, tretinoin was reported to inhibit $\mathrm{CRC}$ cell growth and metastasis in an early study (23). Indeed, Adachi et al found that tretinoin could inhibit cell invasion in human colon cancer (24). Tretinoin can not only treat existing diseases (LMCT), but also serves as a preventive measure for high-risk patients (PMCT). All of the aforementioned drugs have a certain effect on cancer or cancer metastasis, demonstrating the power of our drug screening. In addition to the drugs proven to have anticancer effects, 25 drugs in the brown module and 12 drugs in the blue module have been less studied in cancer-related research based on our literature mining. These drugs also play a role in regulating the brown module or the blue module, similar to the proven drugs. Our results suggest that these yet unproven drugs have the potential to become novel drugs against CRC liver metastasis.

Notably, these drugs all had several targeted genes in the two modules. The major targets in the brown module included the serotonin 5-HT $1 \mathrm{D} \alpha$ autoreceptor gene (HTR1D) and the $\alpha 1$-adrenergic receptor (ADRA1D). Serotonin can stimulate the growth of invasive tumors and carcinoids through the 5-HT receptor, and serotonin receptor antagonists have been shown to successfully prevent cancer cell growth (25). Lamm et al reported that the growth and invasion of human pancreatic cancer cells was suppressed after the downregulation of HTR1D (26). However, the role of HTR1D in CRC remains unclear. We found that HTR1D expression constantly increased during the progression of CRC until the development of liver metastasis. This finding suggests that HTR1D may promote colorectal cancer liver metastasis. Additionally, a study indicated that ADRA1D is closely associated with tumors and participates in the induction of the prostate cancer cell proliferation process (27). However, a role for ADRA1D in $\mathrm{CRC}$ has not been reported. These results suggest that HTR1D and ADRA1D have the potential to become new targets for the treatment of CRC liver metastasis. In the blue module, 5-hydroxytryptamine receptor 2B (HTR2B) and retinoid X receptor $\beta(\mathrm{RXRB})$ are the major drug targets. Zhang et al found that HTR2B was the most significantly altered gene in samples of liver metastases of a uveal melanoma (28). Soll et al found that HTR2B expression was significantly correlated with liver cancer cell proliferation (29). Moreover, RXRB has been shown to promote cell proliferation and survival of triple-negative breast cancer and increased lymph node metastases, tumor recurrence, distant metastasis, and poor prognosis of oral cancer $(30,31)$. These results suggest that HTR2B and RXRB may play major roles in the process of tumor development and metastasis; however, their relationship with CRC liver metastasis has not been elucidated. Our results indicate a close association between HTR2B and RXRB and the progression stage of colorectal cancer liver metastasis. Therefore, these two genes may become new targets for the early prevention and treatment of CRC liver metastasis.

In addition to protein-coding genes, the role of non-coding genes in disease is also important. As shown in Figs. 2 and 3, genes in the yellow and turquoise modules included coding genes and miRNAs that were gradually downregulated during $\mathrm{CRC}$ liver metastasis progression. According to the principle of targeted gene suppression by miRNAs, the inhibitory effect of the downregulated miRNA on the target gene was reduced, resulting in high expression of the target gene. Therefore, gradually downregulating the miRNAs in the yellow and turquoise modules might gradually increase target gene expression and thus contribute to the progression and metastasis of CRC. The yellow module contained two miRNAs. GO analysis revealed that the main function of these miRNAs was in the regulation of the transforming growth factor- $\beta$ (TGF- $\beta$ ) receptor. Are $e t$ $a l$ found that activated TGF- $\beta$ inhibited colorectal cancer liver metastasis, whereas restoration of TGF- $\beta$ receptor II activity reduced the metastasis rate (32). Therefore, these miRNAs negatively regulated the TGF- $\beta$ receptor, possibly promoting the development of colorectal liver metastasis. The turquoise module contained 11 miRNAs that were closely related to cell motion and cell adhesion based on the GO analysis. Clearly, these miRNAs are implicated in tumor cell metastasis because the enhancement of cell migration and reduction of cell adhesion could promote tumor metastasis. Among the 13 miRNAs in the two modules, miR-455 was shown to suppress the proliferation and invasion of CRC cells (33). Wu et al and Zhang et al observed the downregulation of miR-204 in CRC, and miR-320d exhibited low expression in $\mathrm{CD}_{133}{ }^{+} \mathrm{CRC}$ stem cells $(34,35)$. Other miRNAs have not been investigated in CRC research. Our results suggest that the gradual downregulation of miRNAs that regulate the TGF- $\beta$ receptor, the miR- 455 , -204 and $-320 d$, likely play a role in CRC liver metastasis.

In conclusion, using WGCNA to analyze the sequencing data, we have identified unique gene modules in CRC liver metastatic tissue that are not present in the non-metastatic CRC. Using analysis of the miRTarbase database to predict the confirmed target genes of miRNAs, we have identified 13 CRC liver metastasis related candidate miRNAs. Furthermore, analyzing the DrugBank database identified 25 and 12 candidate drugs from the brown and blue modules, respectively, that could potentially block the metastatic processes of the 
primary tumor and inhibit the progression of metastatic tumor in the liver. Data generated from this study not only enhances our understanding of the genetic alterations that drive the metastatic process, but could also guide the development of molecular-targeted therapy for CRC liver metastasis.

\section{Acknowledgements}

This study was supported by Harbin Medical University Postgraduate Innovative Research Projects (grant no. YJSCX2015-19HYD).

\section{References}

1. Jemal A, Bray F, Center MM, Ferlay J, Ward E and Forman D Global cancer statistics. CA Cancer J Clin 61: 69-90, 2011.

2. Wiseman M: The second World Cancer Research Fund/American Institute for Cancer Research expert report. Food, nutrition, physical activity, and the prevention of cancer: A global perspective. Proc Nutr Soc 67: 253-256, 2008.

3. Pasetto LM, Jirillo A, Iadicicco G, Rossi E, Paris MK and Monfardini S: FOLFOX versus FOLFIRI: A comparison of regimens in the treatment of colorectal cancer metastases. Anticancer Res 25B: 563-576, 2005.

4. Zhu D, Ren L and Xu J: Interpretation of guidelines for the diagnosis and comprehensive treatment of colorectal cancer liver metastases in China (v2013). Zhonghua Wei Chang Wai Ke Za Zhi 17: 525-529, 2014 (In Chinese).

5. Steeg PS: Tumor metastasis: Mechanistic insights and clinical challenges. Nat Med 12: 895-904, 2006.

6. Akgül Ö, Çetinkaya E, Ersöz Ş and Tez M: Role of surgery in colorectal cancer liver metastases. World J Gastroenterol 20 : 6113-6122, 2014

7. Postriganova N, Kazaryan AM, Rosok BI, Fretland A Barkhatov L and Edwin B: Margin status after laparoscopic resection of colorectal liver metastases: does a narrow resection margin have an influence on survival and local recurrence? HPB (Oxford) 16: 822-829, 2014.

8. Pan JG, Liu M and Zhou X: Relationship between lower urinary tract symptoms and metabolic syndrome in a Chinese male population. J Endocrinol Invest 37: 339-344, 2014.

9. Simpson AL, Leal JN, Pugalenthi A, Allen PJ, DeMatteo RP, Fong Y, Gönen M, Jarnagin WR, Kingham TP, Miga MI, et al: Chemotherapy-induced splenic volume increase is independently associated with major complications after hepatic resection for metastatic colorectal cancer. J Am Coll Surg 220: 271-280, 2015.

10. Shimada Y: Chemotherapy and molecular-targeted treatment for unresectable hepatic metastases: A Japanese perspective. J Hepatobiliary Pancreat Sci 19: 515-522, 2012.

11. Wang PI and Marcotte EM: It's the machine that matters: Predicting gene function and phenotype from protein networks. J Proteomics 73: 2277-2289, 2010.

12. Hedges SB: The origin and evolution of model organisms. Nat Rev Genet 3: 838-849, 2002.

13. Amrine KC, Blanco-Ulate B and Cantu D: Discovery of core biotic stress responsive genes in Arabidopsis by weighted gene co-expression network analysis. PLoS One 10: e0118731, 2015.

14. Law V, Knox C, Djoumbou Y, Jewison T, Guo AC, Liu Y, Maciejewski A, Arndt D, Wilson M, Neveu V, et al: DrugBank 4.0: Shedding new light on drug metabolism. Nucleic Acids Res 42 (D1): D1091-D1097, 2014.

15. Song WM and Zhang B: Multiscale Embedded Gene Co-expression Network Analysis. PLOS Comput Biol 11: e1004574, 2015.

16. Tang X, Jin R, Qu G, Wang X,LiZ, Yuan Z,Zhao C, Siwko S, Shi T, Wang P, et al: GPR116, an adhesion G-protein-coupled receptor, promotes breast cancer metastasis via the Gaq-p63RhoGEF-Rho GTPase pathway. Cancer Res 73: 6206-6218, 2013.
17. Yan M, Li H, Zhu M, Zhao F, Zhang L, Chen T, Jiang G, Xie H, Cui Y, Yao M, et al: G protein-coupled receptor 87 (GPR87) promotes the growth and metastasis of CD133(+) cancer stem-like cells in hepatocellular carcinoma. PLoS One 8: e61056, 2013.

18. Kim KB, Yi JS, Nguyen N, Lee JH, Kwon YC, Ahn BY, Cho H, Kim YK, Yoo HJ, Lee JS, et al: Cell-surface receptor for complement component $\mathrm{Clq}(\mathrm{gClqR})$ is a key regulator for lamellipodia formation and cancer metastasis. J Biol Chem 286: 23093-23101, 2011.

19. Guo QH, Yang HJ and Wang SD: Olanzapine inhibits the proliferation and induces the differentiation of glioma stem-like cells through modulating the Wnt signaling pathway in vitro. Eur Rev Med Pharmacol Sci 19: 2406-2415, 2015.

20. Lee WY, Lee WT, Cheng $\mathrm{CH}$, Chen $\mathrm{KC}$, Chou CM, Chung $\mathrm{CH}$, Sun MS, Cheng HW, Ho MN and Lin CW: Repositioning antipsychotic chlorpromazine for treating colorectal cancer by inhibiting sirtuin 1. Oncotarget 6: 27580-27595, 2015.

21. Grisoli F, Vincentelli F, Foa J, Lavail G and Salamon G: Effect of bromocriptine on brain metastasis in breast cancer. Lancet 2 : 745-746, 1981.

22. Lissoni P, Vaghi M, Villa S, Bodraska A, Cerizza L, Tancini G and Gardani GS: Antiprolactinemic approach in the treatment of metastatic breast cancer: A phase II study of polyneuroendocrine therapy with LHRH-analogue, tamoxifen and the long-acting antiprolactinemic drug cabergoline. Anticancer Res 23B: 733-736, 2003.

23. O'Dwyer PJ, Ravikumar TS, McCabe DP and Steele G Jr: Effect of 13-cis-retinoic acid on tumor prevention, tumor growth, and metastasis in experimental colon cancer. J Surg Res 43: 550-557, 1987.

24. Adachi Y, Itoh F, Yamamoto H, Iku S, Matsuno K, Arimura Y and Imai K: Retinoic acids reduce matrilysin (matrix metalloproteinase 7) and inhibit tumor cell invasion in human colon cancer. Tumour Biol 22: 247-253, 2001.

25. Sarrouilhe D, Clarhaut J, Defamie N and Mesnil M: Serotonin and cancer: What is the link? Curr Mol Med 15: 62-77, 2015.

26. Lamm V, Hara H, Mammen A, Dhaliwal D and Cooper DK: Corneal blindness and xenotransplantation. Xenotransplantation 21: 99-114, 2014.

27. Morelli MB, Amantini C, Nabissi M, Liberati S, Cardinali C, Farfariello V, Tomassoni D, Quaglia W, Piergentili A, Bonifazi A, et al: Cross-talk between alpha1D-adrenoceptors and transient receptor potential vanilloid type 1 triggers prostate cancer cell proliferation. BMC Cancer 14: 921, 2014.

28. Zhang Y, Yang Y, Chen L and Zhang J: Expression analysis of genes and pathways associated with liver metastases of the uveal melanoma. BMC Med Genet 15: 29, 2014.

29. Soll C, Jang JH, Riener MO, Moritz W, Wild PJ, Graf R and Clavien PA: Serotonin promotes tumor growth in human hepatocellular cancer. Hepatology 51: 1244-1254, 2010.

30. Liu RZ, Graham K, Glubrecht DD, Lai R, Mackey JR and Godbout R: A fatty acid-binding protein $7 / R \times R \beta$ pathway enhances survival and proliferation in triple-negative breast cancer. J Pathol 228: 310-321, 2012.

31. Peng CH, Jiang YZ, Tai AS, Liu CB, Peng SC, Liao CT, Yen TC and Hsieh WP: Causal inference of gene regulation with subnetwork assembly from genetical genomics data. Nucleic Acids Res 42: 2803-2819, 2014

32. Are C, Simms N, Rajput A and Brattain M: The role of transforming growth factor-beta in suppression of hepatic metastasis from colon cancer. HPB (Oxford) 12: 498-506, 2010.

33. Chai J, Wang S, Han D, Dong W, Xie C and Guo H: MicroRNA-455 inhibits proliferation and invasion of colorectal cancer by targeting RAF proto-oncogene serine/threonine-protein kinase. Tumour Biol 36: 1313-1321, 2015.

34. Wu K, He Y, Li G and Peng J: Expression and proliferative regulation of miR-204 related to mitochondrial transcription factor A in colon cancer. Zhonghua Wei Chang Wai Ke Za Zhi 18: 1041-1046, 2015 (In Chinese).

35. Zhang H, Li W, Nan F, Ren F, Wang H, Xu Y and Zhang F: MicroRNA expression profile of colon cancer stem-like cells in HT29 adenocarcinoma cell line. Biochem Biophys Res Commun 404: 273-278, 2011. 